\title{
A New Approach for Alzheimer's Disease Diagnosis by using Association Rule over PET Images
}

\author{
A. Veeramuthu \\ Research Scholar, Dept. of IT \\ Sathyabama University \\ Chennai, India
}

\author{
S.Meenakshi, Ph. D \\ Prof. \& Head, Department of IT \\ SRR Engineering College \\ Chennai, India
}

\author{
P.S. Manjusha \\ PG Student, Department of IT \\ Sathyabama University \\ Chennai, India
}

\begin{abstract}
Alzheimer's disease is usually diagnosed from patient history and clinical information. Finding appropriate technologies and early detection of $\mathrm{AD}$ is of fundamental importance for early treatments. A set of PET images is selected for the study. In order to ensure that a given voxels in different images are refer to the same position the images are normalized using Spatial Normalization which are subjected to noise filter using Butter worth Filter. Intensity Normalization is required to perform direct image comparisons in which the intensity is normalized to an Imax value. Based on Activation Estimation the Region of Interest (ROI) is achieved which are subjected to Association Rule Mining by specifying the minimum support and the confidence values. Finally Computer Aided Diagnosis (CAD) method performs the image classification with verified rules based on threshold. The comparison of previous methods is performed the early finding of $\mathrm{AD}$.
\end{abstract}

\section{Keywords}

Positron Emission Tomography, Spatial Normalization, Intensity Normalization, Region of Interest, Association Rule Mining, CAD.

\section{INTRODUCTION}

Dementia is a serious loss of global cognitive ability which is one of the most severe disorders in the aged population. It is not a single disease (i.e. set of signs and symptoms) which affects the cognitive area such as memory, attention, learning and problem solving. This disease is seem to be increasing particularly in United States, Europe and Japan were more than 5 million people are affected by the disease. In 2013, an estimation of 450,000 people died due to this disease. Neuro imaging is a to mographic technique which refers to brain imaging by sections, through a kind of penetrating wave. At research level, Functional and Structural Neuro imaging are distinctive methodologies. Functional Neuro imaging is used to diagnose metabolic disease such as Alzheimer's disease which includes common methods such as SPECT and PET.

Single Photon Emission Computed Tomography (SPECT) provides clinical information about regional cerebral blood flow (rCBF) and Positron Emission Tomography (PET) measures glucose metabolism rate with the tracer fluorodeoxyglucose. In Alzheimer's patients, the brain regions shows glucose metabolism rate decreased in the temporal and parietal lobs. A normal brain uptakes an average of $20 \%$ of glucose from body where as in Alzheimer's brain, the disease leads to nerve cells death and tissue loss which affects the functioning of brain therefore the uptake of glucose is decreased to $10 \%$ than the normal brain.

The functional images are evaluated through visual assessment by experts who are prone to errors such as manual reorientation, visually reading of tomographic slices. Existing methods for the diagnosis of $\mathrm{AD}$ are based on PCA compression with Support Vector Machine (SVM) classifier, Non-negative Matrix Factorization and Eigen brains with SVM classifier. Development of CAD tool is required to make decision about the presence of abnormalities. The upcoming research has proved that the techniques on machine learning is a dominant analysis tool for brain imaging which includes like Association Rule Learning, Support vector machines for classification, Artificial Neural Network and Bayesian Network. The recent works includes classification based on SPECT, PET or MRI image analysis.

Association Rule (AR) mining is popular and is still research challenge in medical imaging. It is used in analyzing data for if/then pattern. Some of the applications were AR play an important part is shopping basket data analysis, product clustering, catalog design, store layout, extraction of knowledge in software engineering metrics, web personalization, text mining etc. In neural network, AR has also been used for reducing various disease dataset while a classification is performed with neural network model. Programmers use association rule mining to build program capable of machine learning. AR mining is also used to construct hierarchical sequence of clusters and in medical decision making process.

In this paper, a computer based diagnosis system is designed for early diagnosis of Alzheimer's disease by means of AR mining over the discriminant areas. Each image is represented by the feature vector, which consisting of activated ROIs are selected using Fisher Discriminant Ratio (FDR) [1][4][6]. Once the discriminant regions are achieved they are subjected to AR mining [1][3][10][11][12] in which the rules are extracted to train a set image. The extracted rules and test images are verified for the classification whether the test image is an Alzheimer patient image or a normal patient image.

This paper is organized as follows: In section 2 discussed about the related works. AR based CAD system which includes feature extraction, training and testing phase of the proposed methodology is represented in section 3 , experimental procedure of the proposed method is discussed in the section 4 , the performance of previous methods is analyzed with the proposed method which is shown in section 5 finally, conclusions and references are represented in section 6 and 7. 

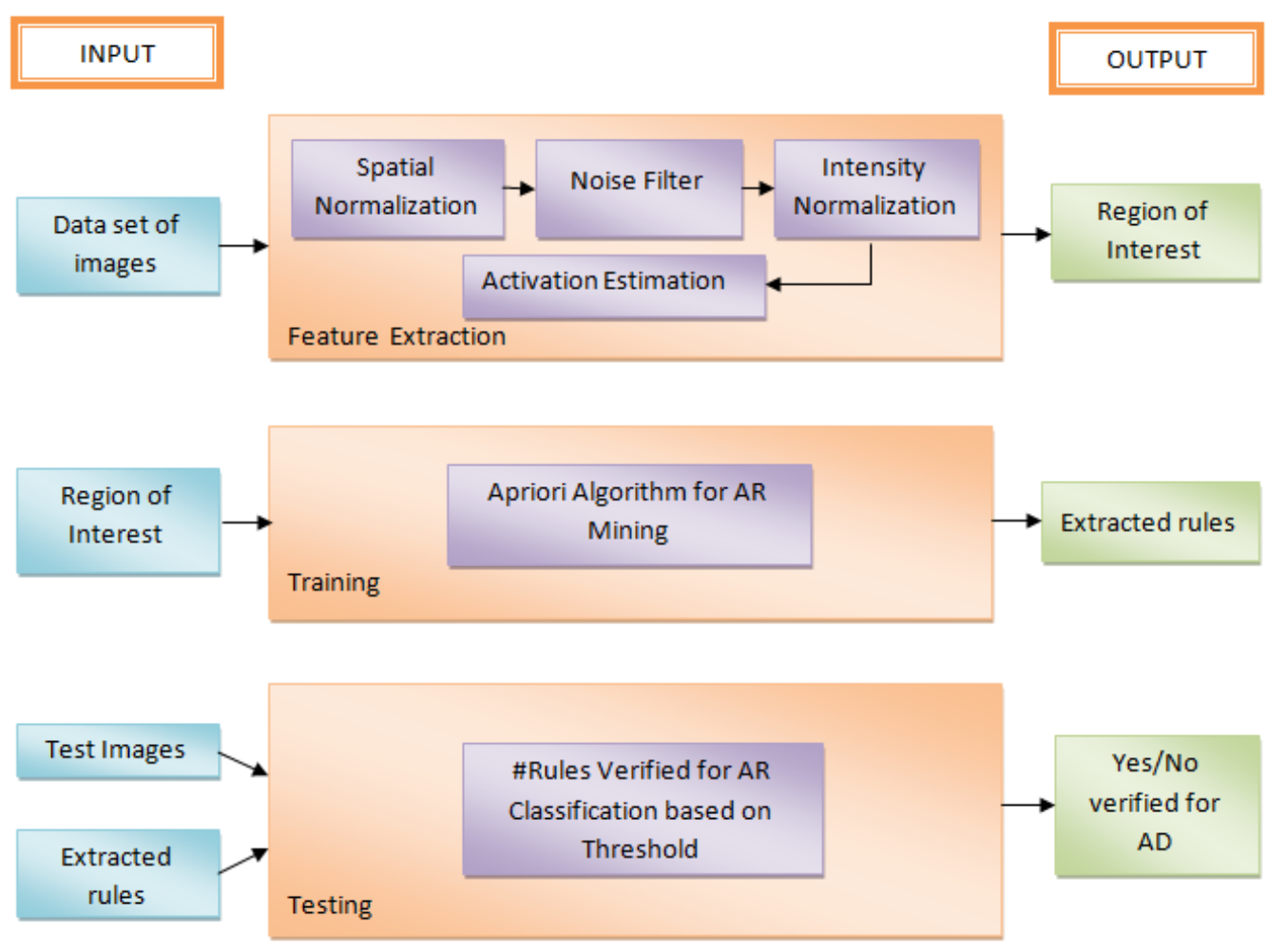

Fig 1: Architecture framework of proposed methodology

\section{RELATED WORK}

The related works includes Voxel as Feature, Principle Component Analysis and Non-negative Matrix Factorization with Support Vector Machines. The component based SVM classification [2][6][8] method uses a set of SPECT images in which, the first phase performs the feature selection and the second phase uses SVM classifier technique.

The FDG PET image analysis [4] method uses feature extraction technique which is a combination of feature space dimension reduction technique and Eigen image based decomposition. The diagnosis based on Eigen brains and support vector machine uses PCA [6] for reduction of dimension for feature space approach and SVM classification technique.

The analysis of functional brain images was also performed using Statistical Parametric Mapping (SPM) [7][8]. A set of brain images are registered in SPM in which the features are selected based on voxel size of the image. The feature selection based on SVM for SPECT images [8] were also performed which uses linear programming formulation method for the classification of image using the spatial normalization information.

The early detection of Alzheimer's disease is performed based on the symptoms of pre-dementia stage (i.e.) mild cognitive impairment [9] which is the study of images with ideal markers on MRI, SPECT, PET and CT scans. Discovering association between different images is a challenge in research work. Fastest algorithms such as Apriori and AprioriTid [10][11] in association rule mining was developed to find the relationship between the transaction in large database. In this paper, Feature extraction based on spatial normalization and selection of discriminant regions using Fisher Discriminant Ratio [1][4][5][6] was proposed.
Association Rule mining algorithm is used for verification of PET images [1][3][10][11][12].

\section{PROPOSED METHODOLOGY}

The focus of proposed work is to verify the test images with the extracted rules based on the given threshold. The proposed framework consists of three phases: (i) Feature Extraction (ii) Training phase (iii) Testing phase are explained as follows.

\subsection{Feature Extraction}

This phase consists of four stages: (i) Spatial normalization (ii) Noise filter (iii) Intensity normalization (iv) Activation estimation. In "Fig.1" shown the architecture framework of proposed methodology.

\subsubsection{Spatial Normalization}

Spatial normalization establishes one-to-one correspondence between the brains of different individuals by matching each image to a standard template. This allows identifying commonalities and differences between groups (e.g. patients' vs. healthy individuals. The PET brain image undergoes spatial normalization process in order to make sure that the given voxels to the same position for maintaining the different images. In "Fig.2", shows the sample PET images. This process is performed using Statistical Parametric Mapping (SPM8) software. The preprocessing steps in SPM8 software consists of realign, normalization, co register, smoothing and segment. The SPM uses Nifti format (neuro imaging format) which produces one $*$.nii file that contains all time point. Alternatively, data can be in *.img and *.hdr format which results in one .img and hdr file per time point. DICOM imported images can also be used in this software.

The normalization warps each individual image into a standard space based on the template. During normalization 
process the affine registration is performed in order to register the set of images. Normalize Estimate and Write is selected which is a direct normalization step. Files are selected for three images which consist of a source image, images to write and template image. The source image is that the image warped to match the templates. The result is set of warps, which can be applied to this image. Images to write are these images are warped according to estimated parameters. They can be any images that are in register with the "source" image. Template image is that images are specified to match the source image. In order to achieve a good registration, the contrast of template must be comparable with that of source image. The default values are chose and the output result is stored with the file name prefix "w" which is shown in "Fig.3", represents normalized PET images.

The affine registration values are displayed for the number of iterations specified during the normalization. In "Fig.5a" and "Fig.5b", represents the graph of affine registration. Thus the images are normalized. In the normalized image the "mm" values are set to 0.0.0 in which the voxels represents the starting point of the scan. Similarly, for the PET brain images of $\mathrm{AD}$ patients are also normalized to acquire a set of normalized images. Therefore from the normalized images the voxels sizes are extracted.

\subsubsection{Noise Filter}

Once the normalized images are retrieved they are subjected to noise filter. The images that are normalized contain some noise therefore they are removed using Butter worth filter. In this type of signal processing filter that is designed for flat frequency response which is possible in pass band. High pass butter worth filter design is used in the proposed method. The "butter" function is designs high pass filter. Butter filters are characterized by a magnitude response that is maximally flat in pass band. The butter worth noise filter is applied using the Matlab function given in equation (1)

$$
[\mathrm{z}, \mathrm{p}, \mathrm{k}]=\operatorname{butter}\left(\mathrm{n}, \mathrm{Wn}, \mathrm{ftype}^{\prime}\right)
$$

For high pass filter the equation (2) is given by,

$$
[\mathrm{z}, \mathrm{p}, \mathrm{k}]=\operatorname{butter}(\mathrm{n}, \mathrm{Wn}, \text { 'high'})^{\prime}
$$

The 'ftype' in the function represents the type of filter (i.e. 'high' for high pass filter) with a normalized cut-off frequency "Wn". Cut-off frequency is that frequency where the magnitude response of filter is $\sqrt{ } 1 / 2$. For butter function, the normalized cut-off frequency Wn must be between 0 and 1 , where 1 is Nyquist frequency, $\pi$ radians per sample.

The zp2sos, which converts a discrete-time zero pole gain representation of the given filter to maintain second order section representation. The Matlab function is given in equation (3),

$$
[\mathrm{sos}, \mathrm{g}]=\mathrm{zp} 2 \operatorname{sos}(\mathrm{z}, \mathrm{p}, \mathrm{k})
$$

The second order section (sos) is attained with gain vector g, which is equivalent to the discrete-time zero-pole-gain filter represented by the arguments $\mathrm{z}, \mathrm{p}$, and $\mathrm{k}$. Vectors like $\mathrm{z}$, and $\mathrm{p}$ contain the zero and poles of the filter's transfer function $\mathrm{H}(\mathrm{z})$, not necessarily in particular order. A difltering object is created which returns a discrete- time and the function in Matlab is given by equation (4),

$$
\text { hd }=\text { dfilt. df2tsos (sos, g) }
$$

Where, hd is the filtering object and $\mathrm{g}$ is the gain for each section. "Fig.4", represents graph of butter worth filter design.

\subsubsection{Intensity Normalization}

After the filtering process, to perform direct image comparison between different images the intensity normalization was required. This intensity of the images was normalized to a value; Imax obtained averaging the $0.1 \%$ of the highest voxels intensities exceeding a threshold. The threshold was fixed to the 10th bin intensity value of a 50-bins intensity histogram, for discarding most low intensity records from outside-brain regions, and preventing image saturation which is shown in "Fig.6a" for intensity normalization graph for n-butter image and "Fig.6b" for intensity normalization graph for a-butter image.

\subsubsection{Activation Estimation}

Selection of discriminant regions is the next process in the feature extraction phase. This process is carried out using Fisher Discriminant Ratio (FDR) in order to find the Region of Interest (ROI) s. This step not only improves accuracy but also reduces computational cost. The voxels, that satisfies particular threshold the greater than 0.2 are selected for further analysis. The regions are achieved by the formula,

$$
\operatorname{FDR}=\left[\left(u_{1}-u_{2}\right) 2 /\left(\text { sigma }_{1}\right) 2+\left(\text { sigma }_{2}\right) 2\right]
$$

Where $u_{i}$ and sigma $_{i}$ denoted that mean value, and the variance for each variable (voxels), respectively. For a given voxels, the ratio grows as the difference of the mean values of each voxels increases, thus being useful to expose discriminant voxels.

For reducing the dimensionality of the problem, the voxels that are above FDR threshold are not selected i.e. the input values are reduced by using AR mining process. Once the discriminant regions are identified, the region of interest (ROI) is achieved using activation estimation. Each image is divided into different blocks. The blocks are activated if the ratio of activated voxels inside is greater than the given threshold. The threshold value is set 0.5 . This threshold-based activation method for block definition provides a good swap between computational complexity and image classification accuracy.

\subsection{Training}

The training phase uses AR mining algorithm in which the extracted activated regions are called as the item sets for the algorithm. The AR mining is an efficient method to find the relationships between different images. The AR mining is defined as follows,

Let $I=\left\{i_{1}, i_{2} \ldots \ldots . i_{m}\right\}$ are extracted from a set of PET images. The algorithm is defined as transaction or implication. A rule is defined as an implication of the form $\mathrm{X} \rightarrow \mathrm{Y}$ where $\mathrm{X}, \mathrm{Y} \subseteq \mathrm{I}$ and $\mathrm{X} \cap \mathrm{Y}=\varnothing$. The support $\operatorname{supp}(\mathrm{X})$ of an item set $\mathrm{X}$ is defined as the proportion of transactions in the data set which contain the item set. The confidence of the rule $\operatorname{conf}(\mathrm{X})$ is defined as in equation (6),

$$
\operatorname{Conf}(\mathrm{X})=\operatorname{Sup}(\mathrm{X} U \mathrm{Y}) / \operatorname{Sup}(\mathrm{X})
$$

\subsection{Testing}

Once the rules are extracted, a test image and extracted rules are subjected to verification for the classification of $\mathrm{AD}$ images based on specified threshold. By checking each image the extracted rules are verified. The image is classified to be normal if the extracted number of verified rules are above the fixed threshold (\# of verified rules $>$ threshold), otherwise the image is classified as AD. 


\section{EXPERIMENTAL RESULT}

\subsection{Data Source}

For the purpose of experiment, a set of PET images which contains normal and $\mathrm{AD}$ affected brain images are used for classification. Existing method, NMF and PCA compression with SVM classifier uses SPECT and MRI dataset.

\subsection{Experimental Procedure}

An data set is chosen for the processing of classification using AR mining. This enters the preprocessing step which performs spatial normalization and intensity normalization process. The feature extraction process takes place in which the region of interest is achieved. The extracted features then enters the training phase where a set of rules (i.e. item sets) are extracted. Finally, test images and rules enters the decision making process in which the verification is performed. If the given image is $\mathrm{AD}$ which said to be true is classified under
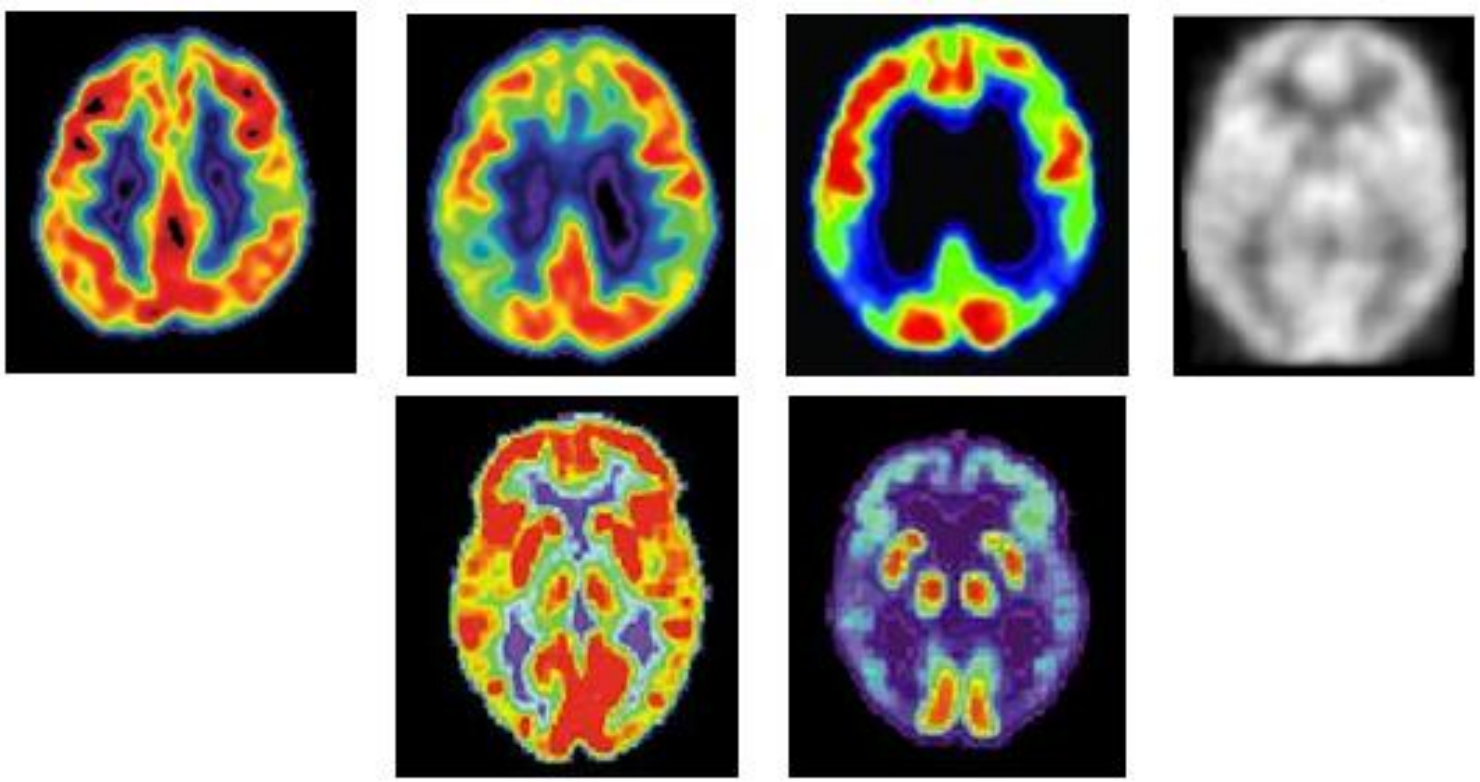

Fig 2: A sample PET image dataset

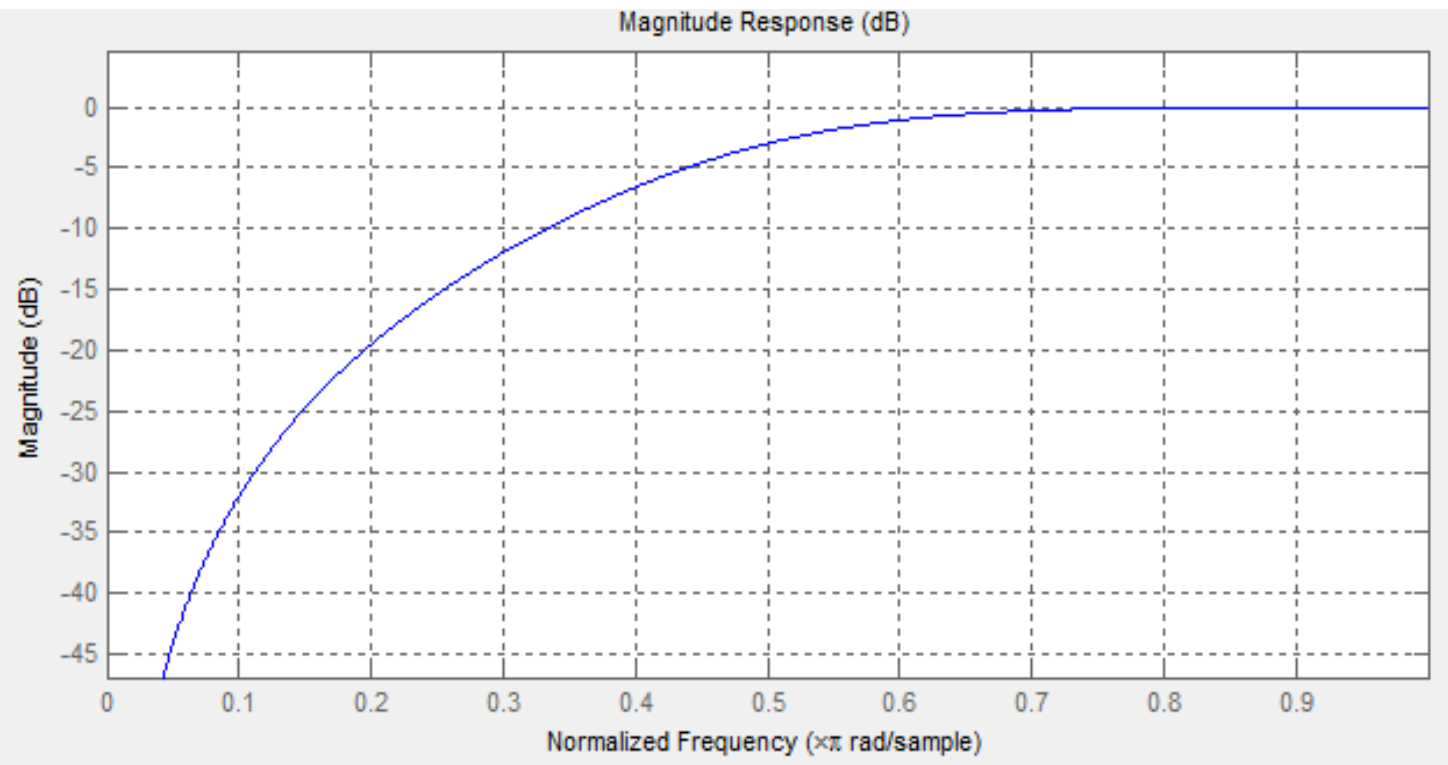

Fig 4: Butter worth filter design graph

$\mathrm{AD}$, else if the given image is normal which said to false is classified under normal. Thus the classification using AR outperforms better than that of previously used method for the diagnosis of $\mathrm{AD}$.

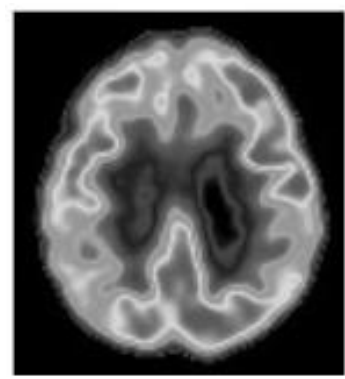

Fig 3: Normalized PET images

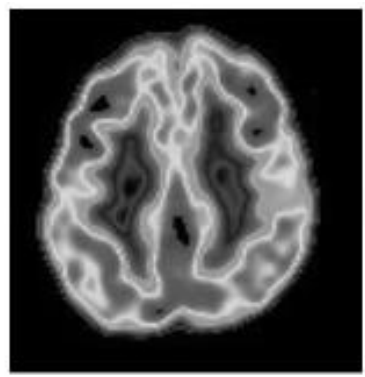




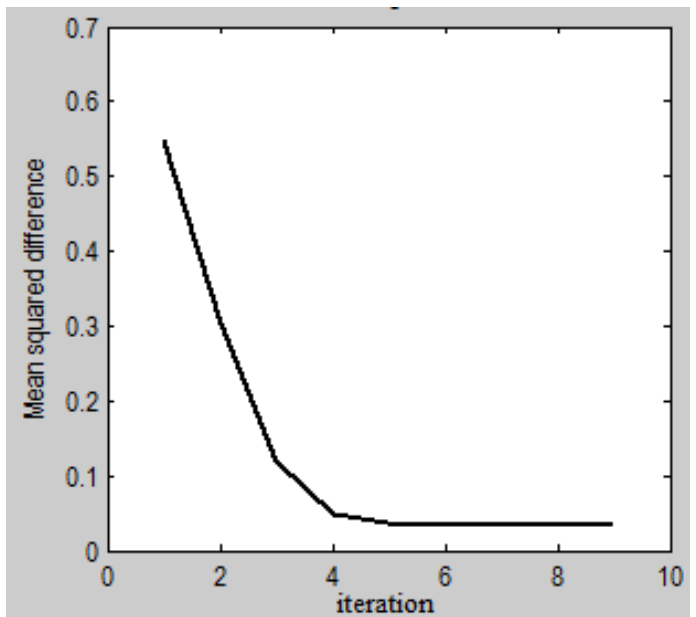

Fig 5a: Affine registration graph
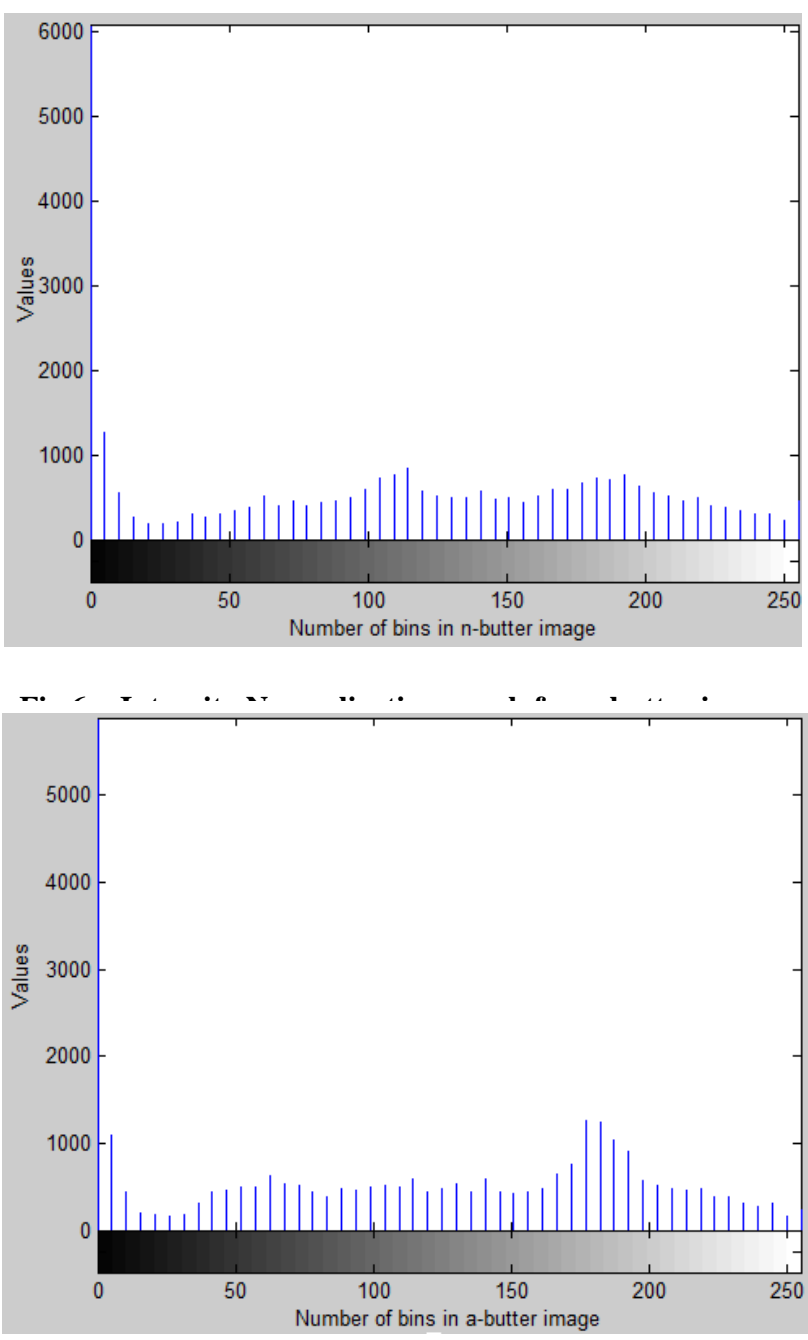

Fig 6b: Intensity Normalization graph for a-butter image

The performance of the proposed method has been improved when compared with existing methods. The accuracy, specificity and sensitivity are more than $90 \%$ for proposed method where as for existing methods such as VAF, PCA with SVM, NMF with SVM ranges from $80 \%$ to $89 \%$. In Table I showed the values for different methods and "Fig.7",

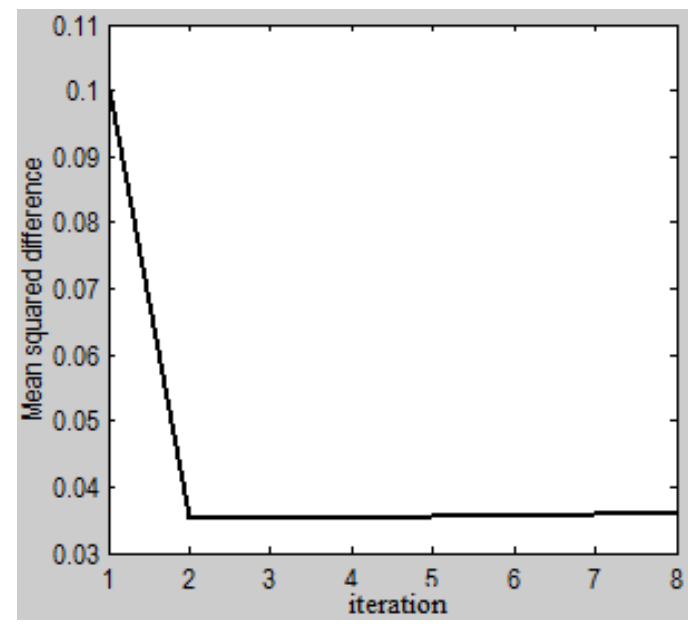

Fig 5h: Affine registration granh

"Fig.8", and "Fig.9" shows the performance comparison graph of previous methods and proposed method.

Table I. Comparison accuracy, specificity, and sensitivity values of previous \& proposed methods

\begin{tabular}{|c|c|c|c|}
\hline Methods & $\begin{array}{c}\text { Accuracy } \\
(\boldsymbol{\%})\end{array}$ & $\begin{array}{c}\text { Specificity } \\
(\mathbf{\%})\end{array}$ & $\begin{array}{c}\text { Sensitivity } \\
(\mathbf{\%})\end{array}$ \\
\hline VAF & 81.18 & 82.60 & 80.74 \\
\hline PCA+SVM & 88.24 & 88.64 & 81.70 \\
\hline NFM+SVM & 84.65 & 85.70 & 79.74 \\
\hline AR & 91.33 & 100 & 82.67 \\
\hline
\end{tabular}

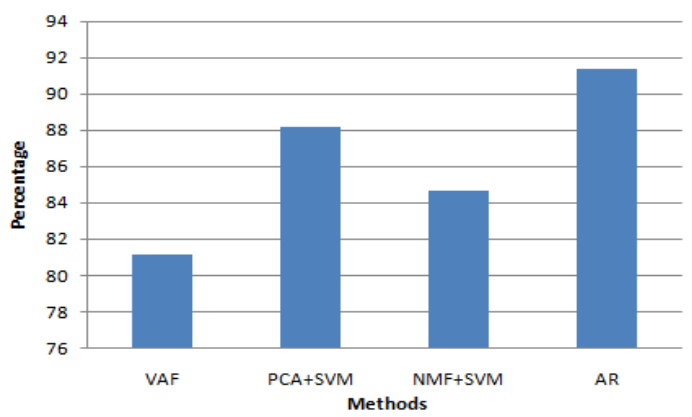

Fig 7: Accuracy

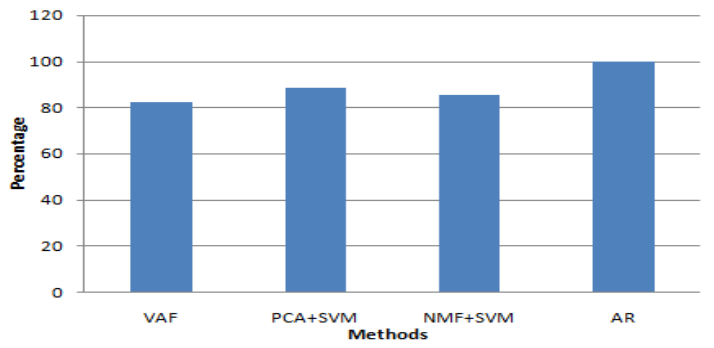

Fig 8: Specificity 


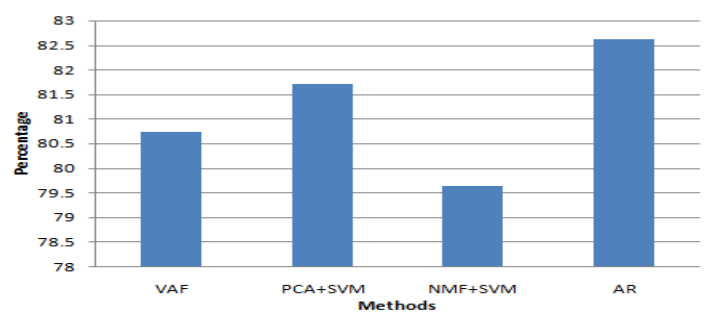

Fig 9: Sensitivity

\section{CONCLUSION}

For classification and design of CAD system, AR was used for the study. Selection of most discriminant regions for further analysis was performed using FDR which has improved the classification result. In this paper feature extraction using preprocessing is performed which is classified using association rule mining algorithm was experimentally evaluated. AR were mined from set of images and the decision making process was formulated were the verification is performed from the extracted rules.

A set of PET images was used for the analysis of CAD system. The accuracy, sensitivity and specificity for AR was high yielding $91.33 \%, 100 \%$ and $82.67 \%$ when compared with previously used methods such as VAF, PCA with SVM, and NMF with SVM. A comparison graph was also presented based on the performance of previous methods.

In future, the work can be carried out with different set of images scans as well as by using different algorithm for classification.

\section{REFERENCES}

[1] R. Chaves , J. Ramirez, J.M. Gorriz, I.A. Illan 'Functional brain image classification using association rules defined over discriminant regions' Dept of Signal theory, Networking and Communication, 2012.

[2] Alvarez, I., M.Gorriz, J., Lopez, M.M., Ramirez, J., Salas-Gonzalez, D., Segovia, F.,Chaves, R., Puntonet, C.G., 2011. Computer aided diagnosis of Alzheimer's disease using component based SVM. Appl. Soft Comput. 11 (2), pp. 2376-2382. 2011.
[3] Chaves, R., Gorriz, J.M., Ramirez, J., Illan, I.A., SalasGonzalez, D., Gomez-R, M, Efficient mining of association rules for the early diagnosis of Alzheimer's disease. Phys. Med. Biol. 56 (18), pp. 6047-6063, 2011.

[4] Alvarez, I., Gorriz, J.M., Ramirez, J., Salas-Gonzalez, D., Lopez, M., Segovia, F., Chaves,R., Gomez-Rio, M., Puntonet, C.G., 18 F-FDG PET imaging analysis for computer aided Alzheimer's diagnosis. Inform. Sci. 181 (4), pp. 903-916, 2010.

[5] Padilla P, Gorriz JM, Ramírez J, Lang EW, Chaves R, Segovia F, López M, Salas-González D, Alvarez.I., Analysis of SPECT Brain Images for the Diagnosis of Alzheimer's Disease Based on NMF for Feature Extraction, Dept of Signal theory, Networking and Communication,2010.

[6] Alvarez, I., Gorriz, J.M., Ramirez, J., Salas-Gonzalez, D., Lopez, M., Puntonet, C.G.,Segovia, F., Alzheimer's diagnosis using eigenbrains and support vectormachines. Electron. Lett. 45 (7), pp. 342-343, 2009.

[7] Friston, K.J., Ashburner, J., Kiebel, S.J., Nicholas.T.E, Penny.W.D.., Statistical Parametric Mapping: The Analysis of Functional Brain Images. Academic Press, 2007.

[8] Fung, G., Stoeckel, J., SVM feature selection for classification of SPECT images of Alzheimer's disease using spatial normalization Knowl. Inform. Syst. 11 (2), pp. 243-258., 2007.

[9] Nestor, P.J., Scheltens, P., Hodges, J.R., Advances in the early detection of Alzheimer's disease. Nat. Rev. Neurosci. 4, pp. S34-S35, 2004.

[10] Agrawal, R., Srikant, R., Fast Algorithms for mining Association rules. In: Int. Conf VLDB Santiago de Chile , Chile, pp. 487-499,1994.

[11] H. Mannila, H. Toivonen, and A. I. verkemo.Efficient algorithms for discovering association rules. In KDD-94: AAAI Workshop on Knoweledege discovery in databases, 1994.

[12] J. R. Quinlan. C4.5: Programs for Machine learning.Morgan Kaufman 1993. 